\title{
Novel Corona Virus COVID-19: An Overview
}

\section{Rohini Agrawal, Prashant Amale, Shilpa A Deshpande*, Priti Bhoyar, Kiran Gomkale and Sunchuwar}

Priyadarshini J. L. Chaturvedi College of Pharmacy, Electronic Zone Building, MIDC, Nagpur, Maharashtra, India

*Corresponding Author: Shilpa A Deshpande, Priyadarshini J. L. Chaturvedi College of Pharmacy, Electronic Zone Building, MIDC, Nagpur, Maharashtra, India.
Received: May 26, 2020

Published: July 30, 2020

(C) All rights are reserved by Shilpa A

Deshpande., et al.

DOI: $10.31080 /$ ASMI.2020.03.0660

\begin{abstract}
Virus Disease of 2019 (COVID-19), was proclaimed as a pandemic emergency by World Health Organisation (WHO) in March 2020. The main causative factor behind the pneumonic viral infection is Severe Acute Respiratoty Syndrome Coronavirus - 2 (SARS-CoV-2) which holds the 75 - 80\% nucleotide sequence similarity with SARS-CoV. Infected and asymptomatic individual are the primary source of human to human transmission due to it become a pandemic, the women with third trimester is also susceptible to this respiratory and pneumonic infection. The time period for the infection is 14 days and the average duration is of 20 days. The infection is clinically manifested mainly by fever, shortness of breath, Acute Respiratory Distress Syndrome (ARDS) etc. Various diagnostic approaches such as Nucleic Acid Amplification Test (NAAT) by using RT-PCR, serological testing etc had been used among which RT-PCR found to be successful in the detection of strain of SARS-CoV-2. Present review focus on the genomic structure of SARS-CoV-2, mechanism, transmission, entry into the host cell, diagnosis, and prevention of the Covid-19. This may be helpful for the development of therapeutic agents used for prophylaxis and treatment of SARS-CoV-2.
\end{abstract}

Keywords: Covid-19; Corona Virus; WHO; SARS-CoV-2; RT-PCR

\section{Introduction}

Corona virus disease (COVID-19); a pneumonic viral infection generated by Severe acute respiratory syndrome coronavirus -2 (SARS-CoV-2) and proclaimed on March 11, 2020 as pandemic by World Health Organisation (WHO) [1]. It is attributed by a respiratory syndrome with a different range of its seriousness from mild upper respiratory illness to severe interstitial pneumonia and acute respiratory distress syndrome (ARDS) [2,3].

\section{Coronavirus}

Coronavirus was first discovered 1960 belongs to the family of Coronaviridae and Nidovirales order with two subfamilies viz.
Orthocoronavirinae and Torovirinae in which Orthocoronavirinae involves four genera $\alpha, \gamma$ and $\delta$-coronavirus [4]. They include total 49 species under the major Riboviria of the suborder of Cornidovirineae [5]. The human coronavirus OC43 (HCoV-OC43), Human coronavirus (HCoV-HKU1), SARS-CoV, SARS-CoV-2 and Middle East Respiratory Syndrome Corona Virus (MERS-CoV) comes under $\beta$-coronavirus. Whereas genus and Human coronavirus NL63 (HCoV-NL63) and Human coronavirus 229E (HCoV-229E) comes under $\alpha$-coronavirus genus. It belongs to the subgenus Sarbecovirus and mostly bears a resemblance with bat coronavirus (96.2\%) [6]. But, intermediary host of transmission is still unknown. 
SARS-CoV-2: Genomic structure and its organisation

\section{Genomic structure}

The globular structure of virus with spike proteins under electron micrographs found to be like a crown structure $[7,8]$. There are numerous types of ORFs, two third part of virus RNA is mainly found in the first Open Reading Frames (ORF) a/b translates two polyproteins, pp1a and pp1ab, and encodes 16 non-structural proteins (NSP) and the rest of them conceal accessory and structural proteins [9-11]. These proteins with other non-structural proteins all together codes within 3' end of genome of virus and 5' two third of viral genome regulates for these non-structural proteins which are essential for the multiplication of virus i.e. RNA-dependent RNA polymerase (RdRP) [12]. Numbers of ORFs present between different protected genes including ORF1ab, spike, envelope, membrane, nucleocapsid and, downstream to the nucleocapsid gene in different coronavirus lineages [4]. It is linked with a nucleoprotein inside the capsid which is incorporated with matrix proteins and encircle of virus possess club shaped projections of glycoprotein while some of the coronavirus also possess Hemagglutinin Esterase protein [13].

Important structural proteins of SARS-CoV-2 and its functions in virus formation

The virus is enclosed with four important structural proteins namely a) Spike Glycoprotein (S) b) small Envelop (E) c) Matrix (M) and Nucleocapsid $(\mathrm{N})$ Protein and hemagglutinin- esterase (HE) protein in some $\beta$-coronaviruses. Among them the three proteins $\mathrm{S} \mathrm{M}$ and $\mathrm{E}$ are submerged in the virus cover. On the other hand the $\mathrm{N}$ protein undergoes interconnection with viral RNA located in the core of particles of virus and helps in the generation of nucleocapsid [14].

S protein: This protein is laboriously glycosylated protein responsible for the formation of homotrimeric spikes on the viral surface and mediates its entry in the host cell [15]. There are three compartments of S protein namely a) large ectodomain, b) a single -pass trans-membrane anchor and c) a short intracellular tail. The huge ecto-domain is composed of two subunits named as S1 and $\mathrm{S} 2$ receptor on $\mathrm{N}$ terminal and $\mathrm{C}$ terminal respectively. Where S1 is a receptor binding subunit and S2 is the membrane fusion unit. These unit shows fusion by two tandem domains, heptad repeats 1 (HR1) and HR2 [16] with crown like structure hence coronavirus is the name given $[17,18]$. The $\mathrm{S} 2$ domain of the $\mathrm{S}$ protein is made up of the fusion protein, a second proteolytic site (S2), through an internal fusion peptide (FP) and two heptad-repeat domains preceding the trans-membrane domain (TM) and internal FP is an similarity between SARS-CoV-2 and SARS-CoV [19].

M Protein: Present abundantly in the structure of virus and responsible to provide shape and with $\mathrm{E}$ protein. It ensures the assembly of virus and leads to the formation of mature virus [20].

E protein: It mainly helps the virus to assemble and release the viral particles in the host cell [20].

N Protein: In the stage of viral assembly these protein helps in the packaging of viral RNA in particles of virus [21,22].

HE protein: This protein is present in some of $\beta$ coronaviruses which shares the similarity with influenza virus hemagglutinin with acetyl esterase activity and can increase the entry of virus in the host cell [23].

\section{Angiotensin converting enzyme 2 (ACE2) receptor}

ACE2 share similarity to ACE and are present in mainly in kidney, heart, and testis [24]. They are also found in respiratory epithelium, type II alveolar cells of lungs, small intestine, and liver" $[25,26]$. ACE2 consists of short intracellular cytoplasmic tail and a longer extracellular domain that contains carboxymonopeptidase activity [27].

\section{Pathophysiology and comparison with SARS-cov-2}

SARS-CoV and SARS-CoV-2 are analogous but novel virus has advanced rate of transmission SRA-CoV. Nsp 2 protein of the novel virus shows modifications which makes virus more contagious [28] and also different ORF8 and ORF10 proteins. It will be interesting and helpful to know about the functions of the proteins. The novel virus are also composed of furin discontinuity site which is absent in SARS-CoV and these may be the rationale behind the increased virulence of the novel SARS-CoV-2.They also share homogenicity in the mechanism of its entry in the host cell [19].

\section{Mechanism of virus entry in the host Cell}

After the cellular entry of virion, the viral glycoprotein $\mathrm{S}$ binds to the host cell receptor ACE2 [29] and gets fused with the host cell membrane with release of viral genome in the cytoplasm [30]. This leads to cleavage of ACE2 and casted by ADAM17 into the extra membrane area and reduction in the level of ACE2. Reduced ACE2 cause alveolar injury and enhances the pulmonary vascular perme- 
ability promoting the conversion of Angiotensin I to Angiotensin II [31]. ORF3a protein is a key for the Calcium ion channel which shares similarities in both virus SARS-CoV and SARS-CoV-2. They interconnect with TRAF3 which enrol the inflammasome complex which is incorporated with NLRP3, ASC and caspase 1 leads to $\mathrm{Ca} 2+$ influx, caspases activation, ROS production and mitochondrial damage which transforms pro -IL $-1 \beta$ to IL- $\beta$ and outcome in the production of cytokines. In addition, the protein ORF8b which is the extended protein of the virus also shows the activation of same path across NLRP3 [32]. Along this same pathway the E protein gives the overstock of cytokines [33]. The combination of all these pathways leads to the cytokines tempest show as the major symptoms of SARS-CoV-2 i.e. respiratory distress [34].

\section{Replication of SARS-CoV-2}

Viral RNA replication is of importance for the surviving of virus in the host cell which is the atypical and evaluative step which helps the virus to reside and grow in the host body cell. The tools that aids for viral replication: a) ORFs b) two replicase genes (rep1a and rep1ab) c) a slippery sequence (5'-UUUAAAC - $\left.3^{\prime}\right)$ d) two polyproteins (pp1a and pp1ab) are most important proteins that contains Nsp proteins (1-11 and 1-16) [35].These Nsp (1/2, 2/3 and 3/4) proteins gather all together and forms a Replicase-Transcriptase Complex (RTC) which makes the environment such as which helps the virus for production and replication of viral RNA. The Nsp12 encodes for RNA dependant RNA polymerase (RdRp) domain where as Nsp13 encodes with RNA helicase domain and RNA 5'-triphosphase, Nsp14 encodes exo-ribonuclease (ExoN) which further favours the viral replication and lastly Nsp16 encrypts 2'-Omethyltransferase activity. Hence, Nsp proteins not only keep the virus alive inside the host cell but also encourage basic synthesis, multiplication and translation [34].

Genomic RNA consists of 5' end region which possess untranslated leader(L) in addition to sequence with the transcription regulation sequence (TRS) located at the downward region of genome. The negative replicase enzyme makes use of negative RNA genome as a template for the formation of small, overlapping messenger RNA (mRNA) molecules. The viral RNA genome enters in the cytoplasm and undergoes translation of polyproteins pp1a and pp1ab [30] which synthesise the non-structural proteins and gives RTC complex and encodes the structural proteins [36]. Moreover, it undergoes translation into the four important structural proteins $\mathrm{S}, \mathrm{N}$, $\mathrm{E}$ and $\mathrm{M}$ which acts as an elementary unit in the synthesis of new viral substances in the host cell, while positive stranded genomic
RNA is utilised as a template to form negative strand. During replication both $\mathrm{S}$ and $\mathrm{N}$ protein joins to the genome and $\mathrm{M}$ protein recruits the endoplasmic reticulum (ER) membrane. These gives rise to the assembly of helical twisted structure of RNA which mature in ER lumen with the help of Nsp proteins. These carries the viral progenies in the host cell membrane with the help of Golgi bodies and are transported in the extracellular space of host cell [37]. These newly formed genomic RNA, nucleocapsid proteins and envelope glycoproteins all gathers an forms the sprouts of virus terminally, virion carrying vesicles attach with plasma membrane and gets released [38].

\section{Transmission of SARS-CoV-2}

This novel virus can be transmitted from animals to animals, animals to humans. The humans to humans especially transmission occurs by coming in close contact with the infected person at a distance of $1-2 m$ and sneezes or coughs with the respiratory droplets may also favour the transmission at a distance of $2 \mathrm{~m}[39,40]$. It also transmit through the objects, fomites or the surfaces [41].

\section{Approaches for the detection of SARS-CoV-2}

- $\quad$ Nucleic acid amplification test (NAAT): These test basically detects the distinctive order viral RNA [42] carried out by RT-PCR method [43]. RT-PCR is designed for the purpose of magnification of targeted DNA molecule in real time and merge reverse transcription of RNA into DNA and PCR amplification of the DNA accompanied to readout using fluorescence [44].

- Test: Assembly of blood samples or respiratory samples collected two times in two weeks apart from nasopharyngeal swab consisting of nasopharyngeal secretions from the back of the nose and posterior pharynx. Swab is mainly a narrow stick made of a short plastic rod that is covered, at one tip, with adsorbing material such as sterile Dacron or rayon and ensuring viral transport media [42].

- $\quad$ Serological test: Paired serum samples may guide the diagnosis of SARS-CoV-2 [42].

- $\quad$ Sequencing of virus: This test involves the detection of genomic mutations of virus [42].

- Chest imaging: These helps to observe the patchy or diffuse asymmetric airspace opacities which shows the homology with the pneumonic symptoms of infected person with SARS-CoV-2 [45] and scans the chest in order to detect the symptoms before it occurs $[46,45]$. 


\section{Conclusion}

WHO had provided guidance regarding the novel infection which is to use the face mask, wash hands properly and completely, regarding the process of disinfection i.e. sanitisation and home medical operating [47].

\section{Bibliography}

1. Wu Joseph T., et al. "Nowcasting and forecasting the potential domestic and international spread of the 2019-nCoV outbreak originating in Wuhan, China: a modelling study". Lancet (London, England) 395.10225 (2020): 689-697.

2. Chen Nanshan., et al. "Epidemiological and clinical characteristics of 99 cases of 2019 novel coronavirus pneumonia in Wuhan, China: a descriptive study". The Lancet 395.10223 (2020): 507-513.

3. Liu Kui., et al. "Clinical characteristics of novel coronavirus cases in tertiary hospitals in Hubei Province". Chinese Medical Journal (2020).

4. Woo Patrick CY., et al. "Coronavirus genomics and bioinformatics analysis". Viruses 2.8 (2010): 1804-1820.

5. Gorbalenya Alexander E. “Severe acute respiratory syndromerelated coronavirus-The species and its viruses, a statement of the Coronavirus Study Group". BioRxiv (2020).

6. Chan Jasper Fuk-Woo., et al. "Genomic characterization of the 2019 novel human-pathogenic coronavirus isolated from a patient with atypical pneumonia after visiting Wuhan". Emerging microbes and Infections 9.1 (2020): 221-236.

7. Bárcena Montserrat., et al. "Cryo-electron tomography of mouse hepatitis virus: insights into the structure of the coronavirion". Proceedings of the National Academy of Sciences 106.2 (2009): 582-587.

8. Neuman Benjamin W., et al. "Supramolecular architecture of severe acute respiratory syndrome coronavirus revealed by electron cryomicroscopy". Journal of Virology 80.16 (2006): 7918-7928.

9. Cui Jie., et al. "Origin and evolution of pathogenic coronaviruses”. Nature Reviews 17.3 (2019): 181-192.

10. Song Zhiqi., et al. "From SARS to MERS, thrusting coronaviruses into the spotlight". Viruses 11.1 (2019): 59.

11. Wu Fan., et al. "A new coronavirus associated with human respiratory disease in China". Nature 579.7798 (2020): 265-269.

12. Masters Paul S. "The molecular biology of coronaviruses". Advances in Virus Research 66 (2006): 193-292.
13. deHaan Cornelis AM., et al. "Coronavirus particle assembly: primary structure requirements of the membrane protein". Journal of Virology 72.8 (1998): 6838-6850.

14. Fehr Anthony R and Stanley Perlman. "Coronaviruses: an overview of their replication and pathogenesis". Coronaviruses. Humana Press, New York, NY (2015): 1-23.

15. Bosch Berend Jan., et al. "The coronavirus spike protein is a class I virus fusion protein: structural and functional characterization of the fusion core complex". Journal of Virology 77.16 (2003): 8801-8811.

16. Xia Shuai., et al. "Fusion mechanism of 2019-nCoV and fusion inhibitors targeting HR1 domain in spike protein". Cellular and Molecular Immunology (2020): 1-3.

17. Yu Fei., et al. "Measures for diagnosing and treating infections by a novel coronavirus responsible for a pneumonia outbreak originating in Wuhan, China". Microbes and Infection (2020).

18. Zumla Alimuddin., et al. "Coronaviruses-drug discovery and therapeutic options". Nature Reviews Drug Discovery 15.5 (2016): 327.

19. Coutard Bruno., et al. "The spike glycoprotein of the new coronavirus 2019-nCoV contains a furin-like cleavage site absent in CoV of the same clade". Antiviral Research 176 (2020): 104742 .

20. Siu YL., et al. "The M, E, and N structural proteins of the severe acute respiratory syndrome coronavirus are required for efficient assembly, trafficking, and release of virus-like particles". Journal of Virology 82.22 (2008): 11318-11330.

21. Chang Chung-ke., et al. "Modular organization of SARS coronavirus nucleocapsid protein". Journal of Biomedical Science 13.1 (2006): 59-72.

22. Hurst Kelley R., et al. "Identification of in vivo-interacting domains of the murine coronavirus nucleocapsid protein". Journal of Virology 83.14 (2009).

23. Klausegger Alfred., et al. "Identification of a coronavirus hemagglutinin-esterase with a substrate specificity different from those of influenza C virus and bovine coronavirus". Journal of Virology 73.5 (1999): 3737-3743.

24. Tipnis Sarah R., et al. "A human homolog of angiotensin-converting enzyme cloning and functional expression as a captopril-insensitive carboxypeptidase". Journal of Biological Chemistry 275.43 (2000): 33238-33243. 
25. Kuba Keiji., et al. "A crucial role of angiotensin converting enzyme 2 (ACE2) in SARS coronavirus-induced lung injury". Nature Medicine 11.8 (2005): 875-879.

26. Paizis Georgina., et al. "Chronic liver injury in rats and humans upregulates the novel enzyme angiotensin converting enzyme 2". Gut 54.12 (2005): 1790-1796.

27. Lambert Daniel W., et al. "Not just angiotensinases: new roles for the angiotensin-converting enzymes". Cellular and Molecular Life Sciences: CMLS 67.1 (2010): 89-98.

28. Angeletti Silvia., et al. "COVID-2019: the role of the nsp2 and nsp3 in its pathogenesis". Journal of Medical Virology (2020).

29. Tortorici M Alejandra and David Veesler. "Structural insights into coronavirus entry". Advances in Virus Research 105 (2019): 93-116.

30. de Wilde Adriaan H., et al. "Host factors in coronavirus replication". Roles of Host Gene and Non-coding RNA Expression in Virus Infection. Springer, Cham, (2017): 1-42.

31. Li Guangdi and Erik De Clercq. "Therapeutic options for the 2019 novel coronavirus (2019-nCoV)". Nature (2020): 149150.

32. Shi Chong-Shan., et al. "SARS-Coronavirus Open Reading Frame-8b triggers intracellular stress pathways and activates NLRP3 inflammasomes". Cell Death Discovery 5.1 (2019): 1-12.

33. Nieto-Torres Jose L., et al. "Severe acute respiratory syndrome coronavirus E protein transports calcium ions and activates the NLRP3 inflammasome". Virology 485 (2015): 330-339.

34. Vellingiri Balachandar., et al. "COVID-19: A promising cure for the global panic". Science of the Total Environment (2020): 138277.

35. Baranov Pavel V., et al. "Programmed ribosomal frameshifting in decoding the SARS-CoV genome”. Virology 332.2 (2005): 498-510.

36. Hussain Snawar., et al. "Identification of novel subgenomic RNAs and noncanonical transcription initiation signals of severe acute respiratory syndrome coronavirus". Journal of Virology 79.9 (2005): 5288-5295.

37. Brian DA and RS Baric. "Coronavirus genome structure and replication". Coronavirus replication and reverse genetics. Springer, Berlin, Heidelberg (2005): 1-30.
38. Guo Yan-Rong., et al. "The origin, transmission and clinical therapies on coronavirus disease 2019 (COVID-19) outbreakan update on the status". Military Medical Research 7.1 (2020): 1-10.

39. Covid CDC., et al. "Coronavirus Disease 2019 in ChildrenUnited States, February 12-April 2, 2020". Morbidity and Mortality Weekly Report 69.14 (2020): 422.

40. COVID, CDC How. Spreads: Centers for Disease Control and Prevention (CDC); 2020 (2020).

41. vanDoremalen Neeltje., et al. "Aerosol and surface stability of SARS-CoV-2 as compared with SARS-CoV-1". New England Journal of Medicine 382.16 (2020): 1564-1567.

42. World Health Organization. Laboratory testing for coronavirus disease ('COVID-19)" in suspected human cases: interim guidance, 19 March 2020. No. WHO/COVID-19/laboratory/2020.5. World Health Organization (2020).

43. Freeman WM., et al. "Quantitative RT-PCR: pitfalls and potential”. BioTechniques 26.1 (1999): 112-22, 124-125.

44. Bustin Stephen A., et al. "The MIQE Guidelines: Minimum Information for Publication of Quantitative Real-Time PCR Experiments". Clinical Chemistry (2009): 611-622.

45. Ai Tao., et al. "Correlation of chest CT and RT-PCR testing in coronavirus disease 2019 (COVID-19) in China: a report of 1014 cases". Radiology (2020): 200642.

46. Salehi Sana., et al. "Coronavirus disease 2019 (COVID-19): a systematic review of imaging findings in 919 patients". American Journal of Roentgenology (2020): 1-7.

47. National Health Commission of People's Republic of China. Guidelines for public protection against novel coronavirus infection.

\section{Assets from publication with us}

- Prompt Acknowledgement after receiving the article

- Thorough Double blinded peer review

- Rapid Publication

- Issue of Publication Certificate

- High visibility of your Published work

Website: www.actascientific.com/

Submit Article: www.actascientific.com/submission.php

Email us: editor@actascientific.com

Contact us: +919182824667 